\title{
Principal Component Analysis of Results Obtained from Finite-Difference Time-Domain Algorithms
}

\author{
José Manuel López-Alonso ${ }^{1}$, José María Rico-García ${ }^{2}, J^{2}$ ier Alda ${ }^{2}$ \\ ${ }^{1}$ College of Optics \& Photonics / CREOL. University of Central Florida. \\ Central Florida Blvd. 4000. Orlando. Florida (USA). 32816-2700 FL \\ ${ }^{2}$ Applied Optics Complutense Group. University Complutense of Madrid. \\ School of Optics. Av. Arcos de Jalón s/n. 28037 Madrid. Spain. \\ Contact e-mail: j.alda@fis.ucm.es
}

\begin{abstract}
Finite-Differences Time-Domain (FDTD) algorithms are well established tools of computational electromagnetism. Because of their practical implementation as computer codes, they are affected by many numerical artefact and noise. In order to obtain better results we propose using Principal Component Analysis (PCA) based on multivariate statistical techniques. The PCA has been successfully used for the analysis of noise and spatial temporal structure in a sequence of images. It allows a straightforward discrimination between the numerical noise and the actual electromagnetic variables, and the quantitative estimation of their respective contributions. Besides, The GDTD results can be filtered to clean the effect of the noise. In this contribution we will show how the method can be applied to several FDTD simulations: the propagation of a pulse in vacuum, the analysis of two-dimensional photonic crystals. In this last case, PCA has revealed hidden electromagnetic structures related to actual modes of the photonic crystal.
\end{abstract}

\section{Introduction:}

One of the most widespread method used to analyze the interaction between the electromagnetic field and the material structures is the so-called Finite-Differences Time Domain (FDTD) method [1]. Its implementation as a numeric algorithm that discretizes space and time into elemental cells for the calculation introduces numerical errors into the solutions: the fields computed step-by-step are affected by numeric artifacts, spurious high-frequency oscillations, and transients induced by the modelization of field sources, interpolation errors, etc [2,3]. When the propagating wavelengths remain unresolved in the grid, the problems are linked to the spatial resolution of the 
computational grid and can be overcome by using a finer computational grid, both temporally and spatially. Another problem has its origin in the finite duration of emitting sources. Here, the Fourier transform of the excitation source adds undesirable frequency components to the original one. Dealing with these artifacts and noise sources is a true challenge in the analysis of the results provided by FDTD. The classification of the artifacts introduced by the numerical noise is an endless task. Their relevance depends on the point of view of the analysis and the purpose of the application. Typically, the methods to filter the effect of the noise are adapted to the given situation, both from the geometrical point of view and from the material properties. Therefore, a careful choice of the excitation source and a proper postprocessing of the information extracted from the FDTD method become a real necessity for some special circumstances. These methods can also be used to improve the quality of the results.

The excitation sources included in FDTD simulations are necessary finite and ought to be discretized in the temporal domain. The finite duration of the temporal window broadens the spectrum of the source, even when it corresponds to a monochromatic stimulus. Then, it could be possible that highfrequency components appear and propagate along the computational grid, adding undesirable contribution to the analysis of the system to a monochromatic excitation. In linear media, these contributions are independent and are superimposed to the expected electromagnetic fields. The result is then contaminated by the high-frequency components. On the other hand the necessary discretization of the temporal and spatial computational grid may introduce sampling artifacts. The effect is an aliased and distorted spectrum. Therefore, the electric and magnetic fields contain unrealistic components generated by the method itself. Several tools have been proposed and used in order to "clean" this noise: smoothing windows [2,4] for the source and application of digital filters to the fields resulting from the computation [5]. These methods reduce considerably the amount of the noise in the final results.

This contribution describes a powerful method to deal with numerical artifacts and noise in FDTD calculations. It is based on a multivariate statistical technique called Principal Component Analysis (PCA) [6]. The method has demonstrated to be very useful when characterizing noise in optical imaging systems [7]. It provides results that can be easily interpreted in terms of images, temporal evolutions, and quantitative contributions to the total variance of the field. One of the advantages of the PCA method is that it can be blindly applied to the output of the FDTD algorithms, without any "a priori” assumption. This avoids any previous adaptation to the practical situation under study. It identifies and classifies spatial-temporal structures that are present in any temporal sequence of spatial distributions of electromagnetic fields. PCA also 
quantifies the contribution of each spatial-temporal structure to the total variance of the original data set. An appropriate grouping of the results of the PCA method makes possible to distinguish those relevant contributions from the noise. Then, PCA becomes a tool to quantify the goodness of the FDTD simulation.

\section{The Finite-Differences Time-Domain Method}

Computational electromagnetism is populated by a great variety of methods and algorithms that try to describe the fundamental interaction between electromagnetic radiation and matter. Among them, one of the most extended and used is the FDTD method. It discretizes the Maxwell equations along time and space and solves in a very clever manner the propagation of the electromagnetic field along the computational grid. The matter is described by the physical constants related with the electric and magnetic field propagation and interaction. Some of the advantages of the method are the easy numeric implementation of the algorithm, the capability to obtain the response of the material and geometric structure along the whole spectrum, it does include any expectation about the form or type of the solution and it does not need any boundary condition when including different kind of materials. Actually, FDTD allows the modelization of homogeneous, inhomogeneous, dispersive, linear, non-linear, isotropic and anisotropic material without changing the inner structure of the algorithm. On the other hand, FDTD is an explicit method, i.e., the fields at a given temporal step are obtained from the fields at the previous temporal step, without any matrix inversion operation. Finally, we may say that FDTD is the preferred in the microwave and millimetre electromagnetic spectrum. Its application to the resolution of structures at optical frequencies is producing a lot of interesting results. However, FDTD also shows some disadvantages that need to be considered: some geometries are hard to fit within a rectangular computational grid. It produces a "staircasing" discretization that may compromise the minimum size of a given spatial feature. On the other hand, FDTD is very demanding with respect to its computer memory needs. However, this fact is being surpassed by the use of more and more powerful computers.

The fundamentals of every computational electromagnetism code are the Maxwell equations. For a homogeneous dielectric, non dispersive and isotropic media without sources, they can be written as follows,

$$
\begin{aligned}
& \vec{\nabla} \cdot \vec{D}=0 \\
& \vec{\nabla} \cdot \vec{B}=0
\end{aligned}
$$




$$
\begin{aligned}
& \partial_{t} \vec{D}=\vec{\nabla} \times \vec{H} \\
& \partial_{t} \vec{B}=-\vec{\nabla} \times \vec{E}
\end{aligned}
$$

FDTD focuses its attention to the rotational equations: the Faraday law, and the Ampere-Maxwell law. This is possible because of the special arrangement of the computational grid that automatically fulfils the divergence equations [1]. This computational grid divides the nodes in the grid depending on the type of field calculated (electric or magnetic) and allows the existence of half-integer index (see figure 1). The algorithm formulated by Yee is based on a "frog-leap scheme" where the values of the electric (magnetic) fields are computed from the values obtained in the previous step and the values obtained for the magnetic (electric) field at the previous step. In the following equation we present the calculation of the $E_{z}$ component from the values of the previous electric field at the same location and the values of the magnetic field, $H_{x}, H_{y}$, at the surrounding locations and in a previous temporal step (just one half of the temporal step),

$$
\begin{aligned}
& E_{z}\left(i-\frac{1}{2}, j+\frac{1}{2}, n+\frac{1}{2}\right)=C_{a} E_{z}\left(i-\frac{1}{2}, j+\frac{1}{2}, n-\frac{1}{2}\right)+ \\
& C_{a} \times\left[H_{y}\left(i, j+\frac{1}{2}, n\right)-H_{y}\left(i-1, j+\frac{1}{2}, n\right)+H_{x}(i-1, j, n)-H_{x}\left(i-\frac{1}{2}, j+1, n\right)\right]
\end{aligned}
$$

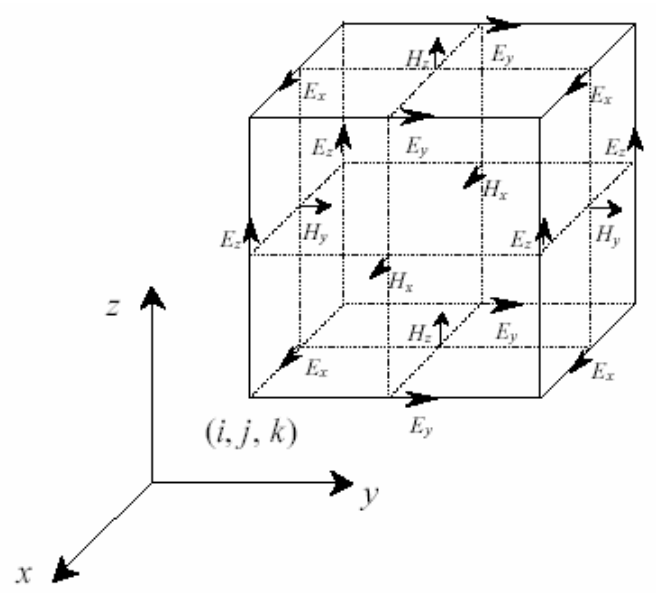

Fig. (1): Structure of the nodes of a unit cell for the implementation of the Yee algorithm. The electric and magnetic fields can be located at integer and halfinteger index values.

The Yee's algorithm keeps the energy constant because the fields do not decay spuriously because of the numeric errors that can be produced along the calculation. However, the stability of the method needs to be assured. This is 
done by adjusting the relation between the values of the spatial and temporal steps [1]. The Courant factor is defined for this purpose in a two dimensional grid as

$$
S=\frac{c \Delta t}{\sqrt{\Delta x^{2}+\Delta y^{2}}},
$$

where the limiting value of this factor is 0.707 . The physical meaning underlying this condition is related with the necessity to have two points in the grid connected by a causality relation. Another source of error in the FDTD method is the numeric dispersion suffered by the field when they travel along the grid. This is caused by the different speed of a monochromatic wave when it travel towards different locations in the grid. This is the so-called numeric anisotropy. This effect can be reduced when the spatial step is diminished $[1,8]$.

FDTD computations use the total field - scattered field zoning of the computational domain [1]. It provides memory savings in simulations and also allows a transparent source to be placed very close to the material structures. In many photonics applications, a pulsed or a continuous plane wave is injected to test the response of the device under study. This is done by means of an interpolating scheme over the limiting surface between the total field zone and the scattered field zone. This interpolation procedure generates noise around the limiting surface in the form of a spurious field. After some time steps, this noise contaminates the rest of the grid. This numerical error has been studied from the point of view of the theory of linear systems and decimated filters [9] have been proposed to reduce the effect.

Summarizing this section we may conclude that FDTD algorithms are adapted very well to the analysis of geometries having different kinds of materials. This is the case of optical antennas, where a metallic structure is coupling the electromagnetic radiation and feed the excited currents towards a rectifying element. On the other hand, the data obtained from the FDTD are snapshots of the electric and magnetic field that can be arranged in a sequence of frames. This type of data is very well prepared to be studied by the PCA method.

\section{The Principal Component Analysis Method}

From a general point of view, the method of principal components analyzes the variance of different observations of a set of variables [6,7]. To apply the method to the characterization of frames produced by FDTD algorithms, we assume that the variables are the frames taken in a time sequence, and the values of the fields at the different locations of the 
computational grid are the observations of these variables. The set of data is arranged as a multidimensional variable denoted as

$$
F=\left\{F_{1}, F_{2}, \ldots, F_{t}, \ldots, F_{N}\right\},
$$

where $N$ is the number of frames and $F_{t}$ is the frame taken at a given time. The whole set comprises all the data produced by the FDTD for the component of the electromagnetic field under study. To apply the principal-component analysis, each frame (corresponding with one of the components of the multidimensional variable $F$ ) is considered a random variable. The realizations of one of these variables are the values of the component of the electromagnetic field at each location of the computational grid. Then the analysis of the covariances between the elements of this set of variables, $F$, is equivalent to calculate the covariance matrix of the frames. The goal of the principal component decomposition applied to FDTD results is to obtain the set of frames as a sum of several processes, showing a clear behavior of their covariances, and presenting a physical meaning of their spatial and temporal evolutions. These processes have to be uncorrelated. To build the covariance matrix of the data we first define the set of variables as in equation (4). Each one of these frames is composed of the signals obtained by the individual pixels. If the two spatial domain of the dimensional grid is discretized having $R$ rows and $C$ columns, it is possible to arrange the $M=R \times C$ values from the twodimensional frame as a column vector. It is important to note that this rearrangement has to be reversible. By use of this method, the set of data is placed in a $M \times N$ matrix, $F$. Before calculating the covariance of the data it is necessary to transform the original frames into a new set of frames having zero mean. This transformation is equivalent to an offset correction that removes a dc level from the signal. The covariance of this set of data is defined by a $N \times N$ matrix. The diagonal elements of $S$ represent the variance of the frames. Meanwhile, the nondiagonal elements are related to the covariance between pairs of frames. The principal component expansion corresponds with new variables, obtained as a linear combination of the original ones, that do not present covariance among them. In addition, the variance of these new variables is arranged in decreasing order. Mathematically, this expansion is obtained by the diagonalization of the $S$ matrix that produces a set of eigenvalues, $\lambda_{\alpha}$, and eigenvectors, $E_{\alpha}$. The diagonalization relation is

$$
\left(S-\lambda_{\alpha} I\right) E_{\alpha}=0 \quad,
$$

where $I$ is the $N \times N$ unity matrix. The set of eigenvectors, $\left\{E_{1}, E_{2}, \ldots, E_{N}\right\}$, can be arranged as a $N \times N$ matrix, $E$, where the column $\alpha$ contains the elements 
of the vector $E_{\alpha}$, obtained from the eigenvalue equation. With this matrix, the principal components are obtained as a $M \times N$ matrix, $Y$, as follows:

$$
Y=\bar{F} E
$$

Where each principal component is given by the following relation:

$$
Y_{\alpha}=\sum_{t} e_{t, \alpha} \overline{F_{t}} .
$$

Even more interesting for the analysis of the FDTD results is the derivation of the original frames in terms of the principal components. The principal components can be taken as spatial distributions of the given electromagnetic field. Therefore, each one of the principal components is a field distribution that is properly combined to produce the original data (see figure 2). This capability makes possible the selection of the significant principal components and the filtering of the undesirable ones. In our case we will be interested in the principal components carrying out information about the evolution of the electromagnetic field and we will like to identify, quantify, and filter the artifacts and noise produced by the algorithm.

The results of the PCA applied to $N$ frames are $N$ eigenvalues $\left(\lambda_{\alpha}\right), N$ eigenvectors $\left(e_{\alpha}\right)$, and $N$ principal components $\left(Y_{\alpha}\right)$. Each principal component is a map having $M$ points. The eigenvectors are orthogonal and represent the temporal evolutions of the contribution of each eigenimage to the original data. When describing frames with a given power spectrum density for a temporalstationary phenomena, PCA can be used to sample the spectrum and characterize it. In that case the involved principal components have quasiharmonic time dependence [10]. Finally, the eigenvalues, explain in decreasing order the contribution of their associated principal component to the total variance of the original data. It is also interesting to note that the capability of the PCA for sectioning the variance and quantify the individual contributions of the obtained principal components to the total variance of the original data, has made possible to reveal spatial-temporal structures hidden behind the main contributions to the variance of the data. In a previous paper we introduce a mechanism to group together a collection of principal components taking into account the uncertainty associated to each eigenvalue [7]. This uncertainty connects two consecutive eigenvalues when their respective uncertainties overlap $[7,11]$. Then, a process is defined as the frames retrieved when only the principal components associated with consecutively overlapped eigenvalues are used. The concept of process is used to ease the interpretation of the results obtained from the PCA (see figure 3 for the case of the analysis of the noise produced by an infrared camera). The classification into processes reduces and 
groups the meaningful principal components and provides an analytical tool that can be automatically applied and implemented.

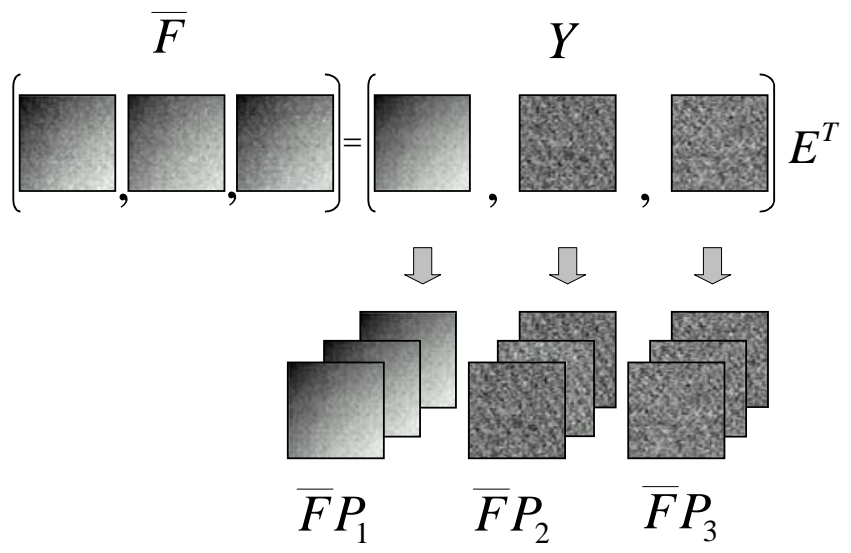

Fig. (2): The original frames (having zero mean) can be retrieved from the principal components obtained by the method. $P_{\alpha}$ are the projectors that allows the transformation from the principal component base to the multidimensional space where the frames $F$ are defined.

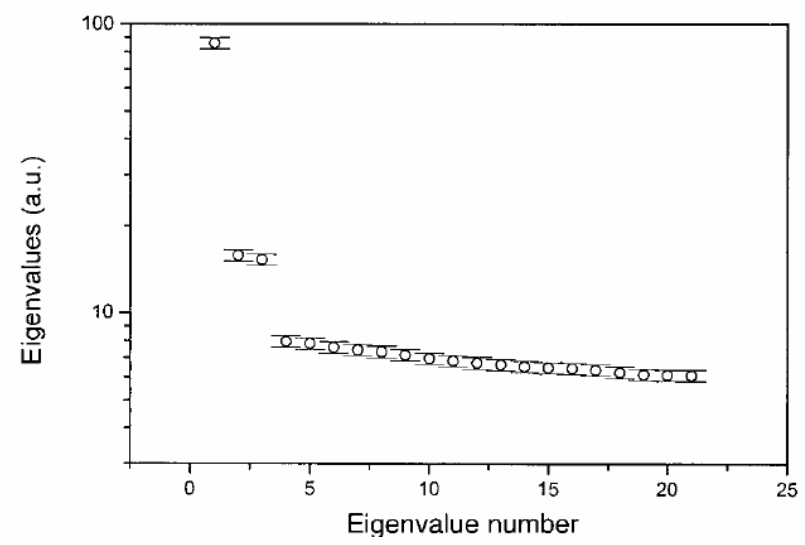

Fig. (3): The eigenvalues in this figure correspond with those obtained in the analysis of the noise of an infrared camera. The concept of process makes possible the identification of three different contributions. The first one corresponds with the highest variance and it is the fixed pattern noise. The third one groupes most of the eigenvalues and it was identified as the temporal noise of the camera. The intermediate one had a characteristic behaviour of a running fringe pattern crossing the image. All these contribution were quantified and filtered out by using PCA.

Summarizing: the principal components, or eigenframes, are spatial distributions with the same units and within the same spatial domain than the original frames. The temporal evolution of a given eigenframe, $Y_{\alpha}$, is described by the associated 
eigenvector, $e_{\alpha}$. The amount of variance explained by the eigenframe is $\lambda_{\alpha}$. Noise is assumed to be a contribution to the data that is independent from the signal, both temporally and spatially. Besides, the level of the noise is usually lower, or even much lower, than the level of the field components calculated in FDTD algorithms. Then, we expect that the first eigenvalues, eigenvectors, and eigenimages, are related with the actual electromagnetic fields, i.e., the signal. Accordingly, the noise should be associated with principal components different from the signal and labelled with greater indices.

The knowledge about the material and geometrical structures, along with the spatial and temporal properties of the excitation, makes possible to identify the results obtained from a FDTD algorithm with good judgment about its validity. This means that we can easily know if the obtained result makes good sense. This is an important issue in the applicability of the PCA method to the results obtained from FDTD. PCA is a blind method that can be used without a prior knowledge of the practical situation. However, when analyzing the outputs of the PCA, the conditions of the simulation and the physical structures under study are necessary to properly understand the results. Besides, some kind of filtering can be used to clean-up the noise and preserve the useful information. PCA gives to this postprocessing analysis the tools to identify those contributions having the largest variance, and therefore representing actual electromagnetic fields. Furthermore, PCA may reveal spatialtemporal structures that could be hidden in the set of data. The capability of the PCA to produce spatial distributions, the eigenimages, is strongly appreciated when analyzing the results. Besides, the spatial-temporal structures related with computational noise, or numeric artifacts are also identified and their contribution is estimated quantitatively. These advantages have been proved successfully in several applications involving the analysis of images [7,10,12-14].

\section{PCA applied to FDTD results}

Now, we are in good condition to apply the PCA to the identification and estimation of numerical noise and artifacts in FDTD algorithms [15]. We will illustrate the method with two significant cases. The first one analyzes the numerical noise associated with the use of the total field - scattered field techniques. In the second case, we have analyzed the confined modes existing in a photonic crystal. In this later case we will check how the location of the excitation point with respect to the symmetry of the computational grid influences on the excited modes, and how the PCA is able to reveal field distributions that are hidden within the data. The geometry of the grid for the calculation is a two-dimensional grid. The electromagnetic fields studied here correspond with $\mathrm{TMz}$ modes, i. e., only $E_{z}, H_{x}$, and $H_{y}$ are non zero. In the following, we will focus our attention on the $E_{z}$ component. 
In the first case, the Total Field / Scattered Field zoning (TF/SF) the FDTD is computed on a two-dimensional square grid having $222 \times 222$ points with a spatial step of $\Delta x=\Delta y=0.025 \mu \mathrm{m}$, and Courant factor of 0.7068 . The source employed to inject energy into the grid is a cosine function having a Gaussian envelope that only is non zero after $t=0$. The pulse has a plane wavefront and the propagation vector is subtending an angle of $60^{\circ}$ with respect to the $Y$ axis. The simulation runs until the pulse travels along the computational grid (see figure 4). A collection of 50 frames is taken during this time at regular intervals. The number of computational temporal steps between frames is 20 .

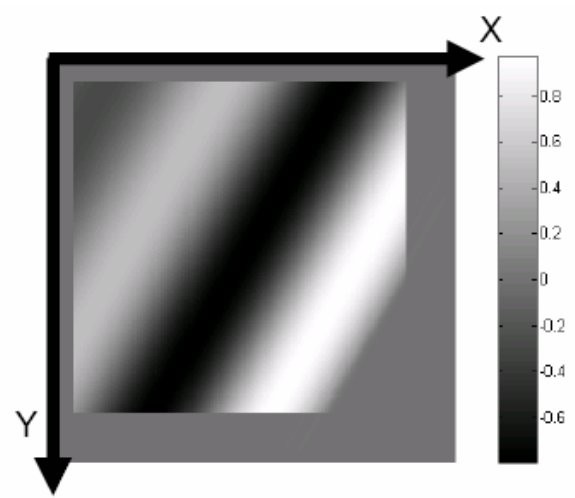

Fig. (4): Map of the electric field obtained for the pulse travelling the computational grid. The limit between the total field (inner square) and the total field is clearly visible in this snapshot. The units are $\mathrm{V} / \mathrm{m}$.

The PCA is directly applied to the data obtained from the FDTD. First of all, we need to transform the original frames into a set having zero mean at each frame. After this, the covariance matrix is calculated and diagonalized to obtain the eigenvalues, the eigenvectors, and the eigenimages (principal components). The first analysis is made on the eigenvalue distribution. This is presented in figure 5. This figure is plotted in linear scale for the first four eigenvalues, that are grouped by pairs. The rest of the eigenvalues are plotted in logarithmic scale. When representing the spatial distribution and the temporal evolution of the two pairs composed by the first four principal components we find that the temporal evolution of the first and second are the same but shifted $\pi / 2$. The same is applicable to the third and fourth principal components (see figure 6). These four contributions represent the $92.44 \%$ of the total variance of the original data. Therefore, we may conclude that these four principal components are carrying out most of the valuable information coming from the original data. Then, the numeric noise and spurious contributions are about $7.56 \%$ of the total variance of the original data. 


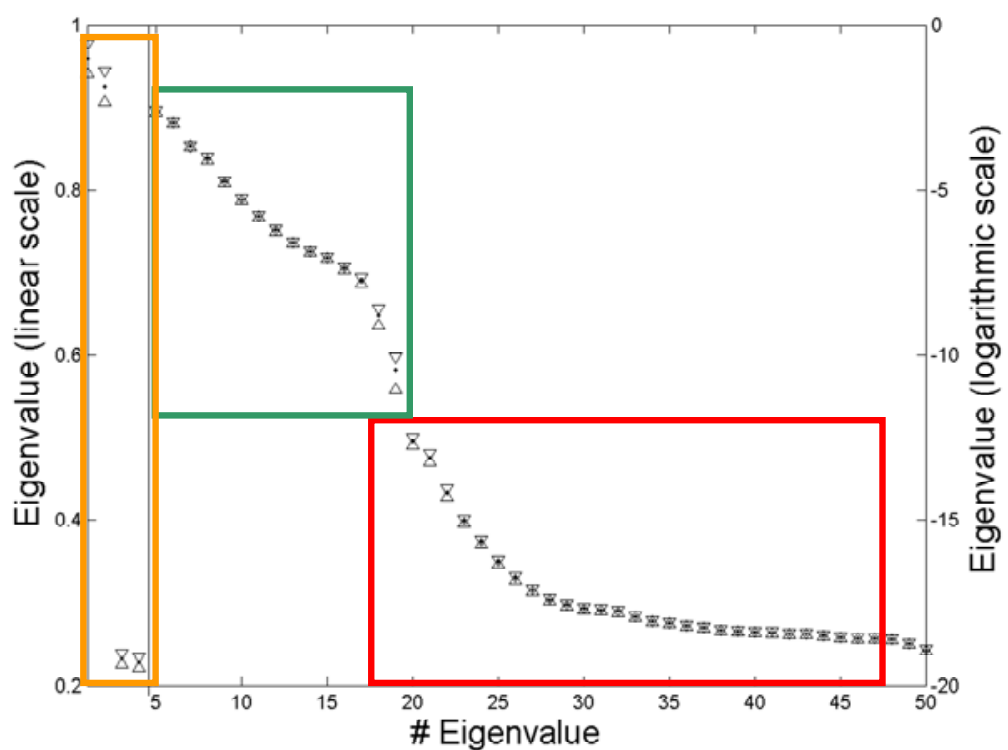

Fig. (5): Plot of the eigenvalues obtained for the propagation of the pulse along the computational grid.

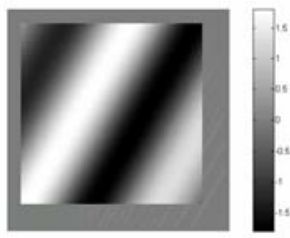

Y1

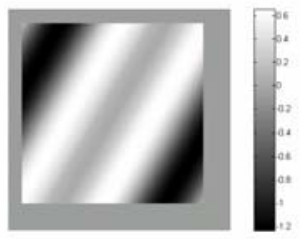

Y3

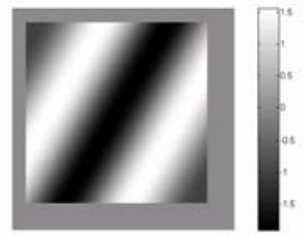

Y2

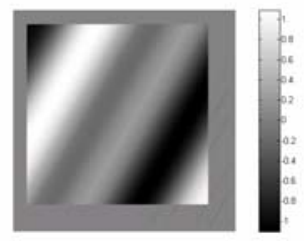

Y4
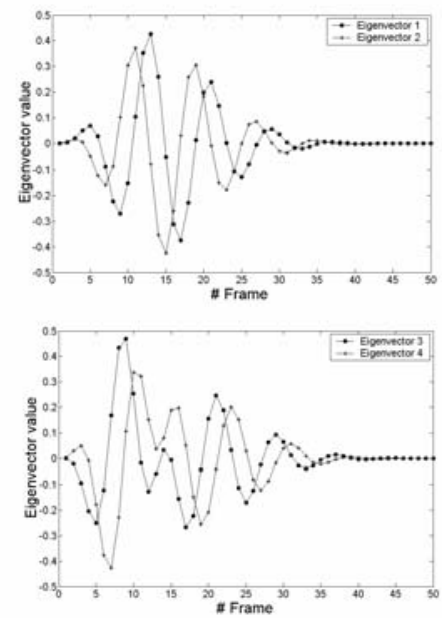

Fig. (6): Representation of the first four principal components (left) and eigenvectors (right) obtained after applying the PCA to the original data obtained from the FDTD algorithm

From the results of this example, we have seen how the PCA method has discriminate the presence of the pulse and it has described the most of propagated electric field by using just four principal components. The other principal components can be identified as noise. Besides, the contribution of the 
different noise processes and types can be estimated allowing the determination of the accuracy of the method, and therefore a quantitative evaluation of the quality of the simulation.

The second example described in this paper is the application of the PCA to the FDTD results obtained for a photonic crystal microcavity formed by dielectric cylinders immersed in air [16]. The central cylinder is different and configures a defect. This defect modifies the energy levels of the photonic crystal and creates electric field distributions that survive within the structure and configure the modes of the microcavity. The selected example corresponds with a lossy microcavity structure. This type of inhomogeneous system has been studied and analyzed in the literature by other methods [5]. The excitation is a soft source located at the center of the structure. This type of source diminishes the effect of the spurious retro-reflections from the source itself. The soft source is "switched off" after 5000 time steps in order to prevent as much as possible the influence of spurious reflections [1,5]. This limitation in the duration of the excitation broadens the spectrum. The grid for implementing the FDTD algorithm is square. We have analyzed two cases, one with an even number of nodes along the grid, $222 \times 222$, and the other with an odd number, $221 \times 221$. The case of an even number of nodes precludes the exact centration of the excitation source and the results should be compared with those obtained from the centered source only possible for the odd number grid. The spatial and temporal steps are the same than those used for the first example. What we want to prove is the influence of the approximate centering of the source that can be obtained with an even number of nodes (decentred case), and the exact centering of the excitation source that can be obtained in the other case (centered case). The $E_{z}$ component is calculated and saved as a snapshot every 10 temporal steps.

The symmetries of the lattice are related with the eigenmodes of the system. An eigenmode is a spatial field distribution whose evolution in time is quasi-harmonic. The eigenmodes of our system have been calculated previously by other authors [5]. To test the influence of the even or odd grid in the eigenmode results we will apply the PCA method to the obtained electric field. The analysis of the eigenframes will reveal the influence of the centering of the source on the results. In figure 7 we have plot the fourth principal component obtained after analyzing the FDTD data obtained for an excitation of the monopolar mode in the case of centered and decentered excitation source. The almost negligible decentering (only one half of the spatial period) produces the excitation of the hexapolar mode. The application of the PCA has revealed the appearance of this spurious artefact that explains only $0.0017 \%$ of the total variance of the original data. 

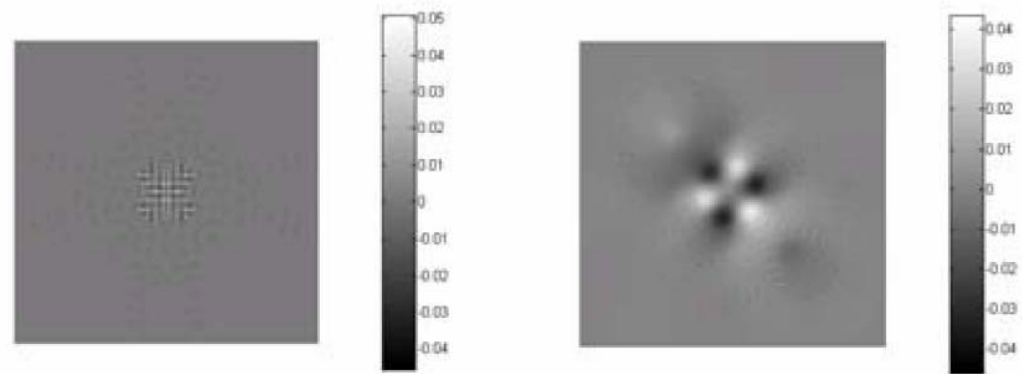

Fig. (7): Map of the electric field for a centered source (left) and a decentered one (right) the decentering is only half of the spatial period.

The first two principal components are not grouped together by the uncertainty of their associated eigenvalues. However, their respective eigenvectors, describing their temporal evolutions, are harmonic functions shifted $\pi / 2$ one with respect to the other. This fact, that was also observed in the previous example has promoted the definition of the so-called quasimonochromatic process. This process groups together those principal components evolving with the same temporal frequency and showing a $\pi / 2$ delay between them. Actually, these principal components can be combined to form a complex principal component having a modulus and a phase. The map of the modulus and phase of the quasi-monochromatic modes can be of help to those researchers dealing with the design and analysis of photonic crystals.

In a recent study, we have applied the PCA to the evaluation of the effect of the fabrication errors on the characteristics of a photonic crystal $[17,18]$. The huge volume of data obtained from the simulation of a large number of statistical realizations of the photonic crystal has been properly analyzed by using the PCA. Some of the results of the electric field distribution are shown in figure 8. In this analyis the PCA could detect the appearance of spurious modes related with the symmetries of the disordered microcavity, even when those contributions were almost negligible. 

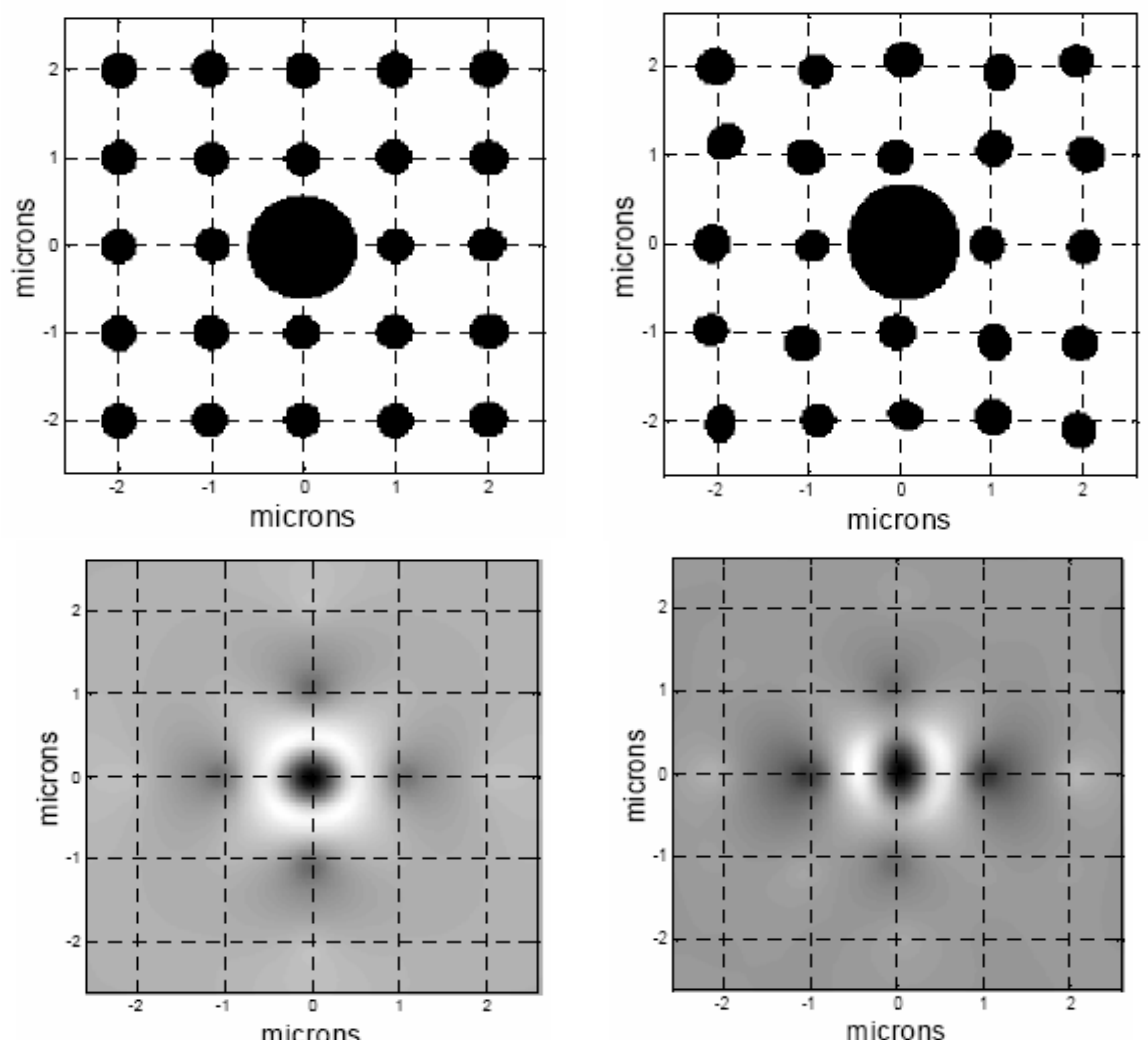

Fig. (8): A snapshot of the electric field distribution (bottom) obtained for a given dielectric permittivity distribution (top) for a given level of manufacture error (left: $1 \%$, and right; $5 \%$ ) is plotted in this figure. The electric field distribution is distorted more for a larger value of the error, as it should be expected.

\section{Conclusions:}

The application of a multivariate statistical technique to the analysis of the output produced by FDTD algorithms has shown its ability to reveal hidden structures of the covariance matrix. Computational electromagnetism is affected by intrinsic deviations due the numeric implementation. The results obtained from FDTD is typically represented as a sequence of frames containing the values of the components of the electromagnetic fields. The input for the PCA method is a sequence of frames, spaced regularly in time, representing a component of the electric or magnetic fields. The results of the PCA are interpreted in terms of the temporal and spatial properties of the electromagnetic 
fields. PCA method produces as many eigenvalues, eigenvectors, and eigenimages as the number of analyzed frames. In a previous contribution we defined a way to group the obtained set of eigenimages into processes. Although the PCA method can be applied to any output of FDTD results, the demonstration of its usefulness for analyzing FDTD data has been illustrated with a couple of examples. The graphical output of the PCA method has made possible an easy and justified interpretation of the results. At the same time, the temporal evolution provided by the eigenvector has been linked to the actual time frequency of the analyzed example with a very good agreement. On the other hand, the PCA method does not need any prior pre-processing of the FDTD data, nor any adaptation of the method itself. It is a blind procedure that can be applied to any temporal sequence of maps of a given electromagnetic field. The outputs of the PCA are analyzed taking into account the knowledge about the geometry and practical realization of the simulated structure and field excitation. This post-processing of the results makes possible to filter the data, to quantify the noise contribution, to identify noise sources and numerical artifacts, and to locate them both spatially and temporally. In this sense, we may say that PCA makes possible to evaluate the quality of FDTD simulations, showing a way to improve them. Finally, we may conclude that PCA method not only provides a way to estimate quantitatively the artifacts induced by FDTD algorithms. It also gives spatial-temporal structures, different from the numerical artifacts, that are expected to appear in actual experiments. The inputs to identify the physical origin of these structures can be found in the temporal evolution given by the eigenvectors and the spatial patterns given by the principal components.

Summarizing, the use of the PCA in the evaluation of the results of FDTD algorithm has been proved successfully and full of advantages with respect to some other approaches. It identifies, quantifies, and maps the electric field distributions explaining a given amount of variance of the data. An appropriate application of the method can be used to filter or enhance some contributions that could be well hidden within the data.

\section{Acknowledgments}

Most of the work presented in this paper has been possible due to the economic support of several research projects. Among them, we would like to acknowledge the participation of the Ministerio de Ciencia y Tecnología of Spain through the project TIC2001-1259, and to the Consejería de Educación of the Comunidad de Madrid (Spain) through the project GR/MAT/0497/2004. The collaboration between the University Complutense of Madrid, and the University of Central Florida has been possible thanks to a collaboration agreement among these two institutions. 


\section{References}

1. Taflove, Computacional Electrodynamics: The Finite-Difference Time Domain Method, 2nd edition, Artech House (2000).

2. L. Gürel and Ugur Ogüz, "Signal-Processing Techniques to Reduce the Sinusoidal Steady-State Error in the FDTD Method", IEEE Trans. Antennas Propagat., 48, 585-593 (2000).

3. A. Taflove and K. Umashankar, "Radar cross section of general threedimensional scatterers”, IEEE Trans. Electromagn. Compat., EMC- 25, 433-440 (1983).

4. V. Oppenheim and R. W. Schafer, Discrete-Time SignalProcessing, Englewood Cliffs, NJ. Prentice-Hall, 1989.

5. S. Guo and S. Albin, "Numerical Techniques for excitation and analysis of defect modes in photonic crystals", Opt. Express, 11, 1080-1089 (2003).

6. D. F. Morrison, Multivariate Statistical Methods, 3rd ed. McGraw-Hill, Singapore, 1990, Chap. 8.

7. J. M. López-Alonso, J. Alda, E. Bernabéu, "Principal components characterization of noise for infrared images”, Appl. Opt., 41, 320-331 (2002).

8. J. S. Juntunen, D. Tsiboukis. "Reduction of numerical dispersion in FDTD method through artificial anisotropy". IEEE Trans. Microwave Theory and Technology 48, 582-588 (2000).

9. U. Ogüz, L. Gürel, and O. Arikan, "An efficient and accurate technique for the incident-wave excitations in the FDTD method", IEEE Trans. Microwave Theory and Technology, 46, 869-882 (1998).

10. J. M. López-Alonso, J. Alda, "Operational parametrization of the $1 / \mathrm{f}$ noise of a sequence of frames by means of the principal components analysis in focal plane arrays”, Optical Engineering, 42, 1915-1922 (2003)

11. R. B. Cattell, "The scree test for the number of factors," J. Multivar. Behav. Res. 1, 245-276 (1966).

12. J. M. López-Alonso, J. Alda, "Bad pixel identification by means of the principal components analysis" Optical Engineering, 41, 2152-2157 (2002)

13. J. M. López-Alonso, J. Alda, "Characterization of dynamic sea scenarios with infrared imagers”, Infrared Physics and Technology, 46, 355-363 (2005)

14. J. M. López-Alonso, J. Alda, "Characterization of artifacts in fully-digital imageacquisition systems. Application to web cameras”, Optical Engineering, 43, 257265 (2004)

15. J. M. López-Alonso, J. M. Rico-García, J. Alda, "Numerical artifacts in finitedifference time-domain algorithms analyzed by means of Principal Components", IEEE Transaction on Antennas and Propagation, 53, 2920-2927 (2005).

16. J. M. López-Alonso, J. M. Rico-García, J. Alda, "Photonic crystal characterization by FDTD and Principal Component Analysis”, Optics Express, 12, 2176-2186 (2004)

17. J. M. Rico-García, J. M. López-Alonso, J. Alda, "Multivariate analysis of photonic crystal microcavities with fabrication defects”, Proceedings SPIE, 5840, 562-571, (2005)

18. J. M. Rico-García, J. M. López-Alonso, J. Alda, "Characterization of Photonic Crystal Microcavities with Manufacture Imperfections”, Optics Express 13, 38023815, (2005) 